\title{
Aortic Adventitial Fibroblasts Participate in Angiotensin-Induced Vascular Wall Inflammation and Remodeling
}

\author{
Brian C. Tieu ${ }^{a, b}$ Xiaoxi Ju ${ }^{a}$ Chang Lee ${ }^{b}$ Hong Sun $^{b}$ Wanda Lejeune ${ }^{b}$ \\ Adrian Recinos, III ${ }^{b} \quad$ Allan R. Brasier ${ }^{a-c}$ Ronald G. Tilton ${ }^{b, c}$ \\ ${ }^{a}$ Departments of Biochemistry and Molecular Biology, ${ }^{\mathrm{b}}$ Division of Endocrinology, Internal Medicine, and \\ 'Sealy Center for Molecular Medicine, University of Texas Medical Branch, Galveston, Tex., USA
}

\section{Key Words}

Aorta $\cdot$ Aortic adventitial fibroblasts $\cdot$ Macrophages $\cdot$

Angiotensin II $\cdot$ Interleukin- $6 \cdot$ MCP-1 $\cdot$ CCR2

\begin{abstract}
Background/Aims: The role of adventitial fibroblasts in the vascular inflammation observed in the adventitia of large vessels in numerous cardiovascular diseases remains unclear. Our objective was to explore the contribution of these cells to angiotensin II (Ang II)-induced aortic inflammation and adventitial expansion. Methods: Cytokine production by primary human aortic adventitial fibroblasts (AoAF) in tissue culture was detected using multiplex ELISA, and increases in cytokine mRNA following Ang II stimulation were quantitated by real-time PCR. The ability of AoAF-derived MCP-1 to attract monocytes was studied in vitro using Boyden assays, and the resulting effect of the monocyte-AoAF interaction on fibroblast proliferation was measured in vitro using proliferation and ${ }^{3} \mathrm{H}$-thymidine incorporation assays. Ang IIinduced fibroblast proliferation was measured in vivo using aortic digestion of single cells followed by flow cytometric quantification of fibroblast numbers as well as fibroblast and PCNA immunostaining. The ability of monocytes to induce
\end{abstract}

AoAF proliferation was demonstrated in vivo using $\mathrm{CCR}^{+/+}$ wild-type monocyte adoptive transfer into Ang II-stimulated CCR2-null mice which can produce MCP-1 but have cells lacking the MCP-1 receptor - CCR2. Results: AoAF constitutively secreted numerous proinflammatory cytokines, particularly IL-6 and MCP-1, whose gene expressions were further upregulated in response to Ang II stimulation. AoAF-derived MCP-1 was potent in recruiting THP-1 monocytes in vitro, and these monocytes stimulated AoAF proliferation based on a flow cytometric assessment of cell number and ${ }^{3} \mathrm{H}$-thymidine incorporation in tissue culture. In vivo, Ang II induced fibroblast proliferation, increased fibroblast and PCNA adventitial staining, and blunted inflammatory responses in the $\mathrm{CCR2}^{-/-}$background. Injection of $\mathrm{CCR2}^{+/+}$monocytes into Ang II-treated CCR2 ${ }^{-1-}$ mice restored adventitial thickening which resulted in increased fibrosis secondary to adventitial fibroblast proliferation. Conclusions: Our results suggest that Ang II-stimulates AoAF to recruit monocytes via fibroblast-derived MCP-1, and the recruited monocytes further activate fibroblast proliferation, adventitial thickening, and additional cytokine production. This fibroblast-monocyte amplification loop may critically mediate hallmarks of adventitial inflammation common to many cardiovascular diseases.

Copyright $\odot 2010$ S. Karger AG, Basel

\section{KARGER}

(c) 2010 S. Karger AG, Basel

Fax +41 613061234 E-Mail karger@karger.ch www.karger.com www.karger.com/jvr
Dr. Ronald G. Tilton

8.138 Medical Research Building

University of Texas Medical Branch

301 University Blvd., Galveston, TX 77555-1060 (USA)

Tel. +1 409772 8738, Fax +1 409772 8709, E-Mail rgtilton@ utmb.edu 


\section{Introduction}

Little is known about the contribution of adventitial fibroblasts to vascular wall inflammation. Their ability to produce collagen and reactive oxygen species (ROS) via $\mathrm{NAD}(\mathrm{P}) \mathrm{H}$ oxidase has been demonstrated $[1,2]$; moreover, in these cells, ROS mediate cell proliferation and differentiation to myofibroblasts [3], which are thought to be major contributors to neointimal thickening after severe endoluminal vascular injury [4-6]. In addition to ROS production, activated fibroblasts assume a secretory phenotype responsible for cytokine secretion. Interleukin-6 (IL-6) is predominantly expressed in the adventitia of $\mathrm{LDLR}^{-/-}$mice infused with angiotensin II (Ang II) [7], and the chemokine MCP-1 has been detected there preceding its expression in the intima following the recruitment of cells expressing CCR2, the receptor for MCP-1, to the vessel wall $[8,9]$. IL- 6 is known to be involved in monocyte activation and MCP-1 functions as a monocyte chemoattractant, so that they work in complementary ways in the coordination of leukocyte recruitment and activation in tissues. Importantly, approaches used in previous studies have not allowed definitive identification of the cell type(s) in the vessel wall responsible for producing these cy tokines and chemokines or their functional roles. Knowledge of the spectrum of cytokines and chemokines produced by adventitial fibroblasts and the role of Ang II in upregulating their expression will be valuable in understanding the role of fibroblasts in adventitial inflammation.

Vascular wall inflammation has been observed in many cardiovascular diseases [10] and involves the cellular infiltration of lymphocytes, plasma cells, macrophages, eosinophils, and mast cells into the adventitial layer of the vessel wall [11-14]. Extensive cellular infiltration into the adventitia is highly correlated with advanced atherosclerotic plaque disruption, which augments the risk of mortality [15-17], while the expression of inflammatory cytokines in the adventitia is strongly correlated with advanced atherosclerosis [18]. Increased adventitial fibrosis also has been observed in atherosclerosis, mechanical injuries from angioplasty, and abdominal aortic aneurysms [19]. A variant of abdominal aortic aneurysms, inflammatory aortic aneurysm, has extensive adventitial infiltration of macrophages and pronounced thickening of the adventitia, which has been attributed to proliferation of adventitial myofibroblasts [20]. Whether cytokines and chemokines produced by adventitial fibroblasts can recruit leukocytes and how their recruitment affects fibroblast proliferation remains unknown.
Ang II is well known to play an important role in cardiovascular diseases such as atherosclerosis and aortic aneurysms and dissection. In vivo and in vitro, it can induce important markers of vascular inflammation, but its effects on the adventitial fibroblasts are not well known. Our focus was on IL-6 and MCP-1 since we report that human aortic adventitial fibroblasts (AoAF) in tissue culture produced these in relatively greater abundance than numerous other cytokines and chemokines. Using a combination of tissue culture and in vivo animal studies, we report that AoAF contribute significantly to vascular inflammation by producing IL- 6 and MCP-1. We demonstrate that the MCP-1 produced by adventitial fibroblasts can recruit monocytes, which, in turn, promotes AoAF proliferation in vitro and in vivo. In addition, we show that Ang II potently induces the expression of IL- 6 and MCP-1.

\section{Methods}

\section{Cell Culture}

Primary cultures of human AoAF (catalog No. CC-7014; Cambrex-Lonza, Walkersville, Md., USA) were purchased, grown in stromal cell growth medium (SCGM) containing 5\% FBS, and used from passages 4 to 9 . These cells were obtained from normal aortic tissue and their identity and purity were confirmed based on morphology, growth characteristics, and negative immunofluorescent staining for smooth muscle $\alpha$-actin. THP-1 cells, i.e. human leukemic promonocytic cells, were obtained from ATCC (Manassas, Va., USA) and cultured in RPMI 1640 medium containing 10\% FBS. Coculture experiments were performed with $50 \times 10^{3}$ AoAF and $200 \times 10^{3}$ THP-1 monocytes in SCGM.

\section{Cell Production of Cytokines and Chemokines}

To determine the constitutive production and secretion of cytokines and chemokines, $50 \times 10^{3}$ AoAF and $200 \times 10^{3}$ THP- 1 monocytic cells were grown in $1 \mathrm{ml}$ of their respective media for $36 \mathrm{~h}$. Twenty-five-microliter samples of fibroblast- or monocyteconditioned medium were assayed in duplicate using BioSource Multiplex kits (Invitrogen, Carlsbad, Calif., USA), and standard curves assayed in parallel were used to determine cytokine concentrations. Data were expressed as means \pm standard deviation (SD) for 6 individual samples for each cell type.

\section{Monocyte Migration Assay}

The migration of THP-1 monocytic cells to AoAF-conditioned medium was assessed using Transwell polycarbonate membrane inserts with $8-\mu \mathrm{m}$ pores (Corning Lifesciences, Lowell, Mass., USA). Briefly, $2 \times 10^{6}$ THP-1 cells in $100 \mu \mathrm{l}$ of serumfree SCGM were placed above the inserts, and $600 \mu \mathrm{l}$ of AoAFconditioned medium or SCGM was placed below. After $2 \mathrm{~h}$ of incubation at $37^{\circ} \mathrm{C}$, the numbers of cells that migrated to the bottom wells were assessed using a Z2 Coulter Counter (Beckman Coulter, Fullerton, Calif., USA). EDTA (10 mM) was used to detach migrated cells still attached to the membrane before centri- 
fuging the plates to collect the migrated cells in the bottom chamber. To confirm that all cells had detached, inserts were stained with $1 \%$ crystal violet in $2 \%$ ethanol to visualize cells. For inhibition studies, neutralizing MCP-1 antibodies (clone 2H5; Biolegend, San Diego, Calif., USA) or Hamster IgG control antibodies (eBioscience, San Diego, Calif., USA) were incubated with AoAFconditioned medium for $30 \mathrm{~min}$ prior to plating.

\section{AoAF Proliferation Assays}

The ability of Ang II and monocytes to induce AoAF proliferation in vitro was assessed in 2 separate experiments. First, AoAF were plated at $50 \times 10^{3}$ cells per well in 12 -well plates and grown in SCGM for $24 \mathrm{~h}$. The medium was replaced with SCM containing $0.25 \% \mathrm{BSA}, 0.2 \% \mathrm{FBS}$, and cells stimulated with $1 \mu \mathrm{M}$ Ang II for $16 \mathrm{~h}$. During the final $4 \mathrm{~h},{ }^{3} \mathrm{H}$-thymidine $(2 \mu \mathrm{Ci} / \mathrm{ml})$ was added. Plates were briefly washed with $2 \mathrm{ml}$ of cold PBS, and then $2 \mathrm{ml}$ of cold $10 \%$ TCA were added and incubated at $4^{\circ} \mathrm{C}$ for $2 \mathrm{~h}$. Cells were washed with $95 \%$ ethanol and allowed to dry for $1 \mathrm{~min}$. Five hundred microliters of $0.2 \mathrm{~N} \mathrm{NaOH}$ was added for $2 \mathrm{~h}$ to dissolve the cells. Four hundred fifty microliters of lysate was added to $5 \mathrm{ml}$ of scintillation cocktail, mixed, and dark-adapted for at least $2 \mathrm{~h}$ before counting in a Geiger counter (Beckman Coulter). In a second similar experiment, AoAF were plated and grown, and the medium was replaced with SCM as described above, but cells were stimulated with $1 \mu \mathrm{M}$ Ang II for 4 days. AoAF were detached in $0.5 \mathrm{ml}$ Accumax and diluted in $9.5 \mathrm{ml}$ of an Isoton II solution for counting with a Z2 Coulter Counter.

In a second series of experiments, AoAF were labeled with $2 \mu \mathrm{M}$ PKH26 (Sigma, St. Louis, Mo., USA) prior to the start of coculture experiments with THP-1 monocytic cells that remained unlabeled. Briefly, AoAF were labeled according to the manufacturer's instructions by resuspending the cells in $1 \mathrm{ml}$ of solution A (used to dilute $\mathrm{PKH} 26$ ); a $4-\mu \mathrm{M}$ solution of $\mathrm{PKH} 26$ dye in $1 \mathrm{ml}$ was made in solution B. Solution A was combined with solution $\mathrm{B}$ and the cells were incubated for $3 \mathrm{~min}$ at room temperature. The final concentration of PKH26 was $2 \mu \mathrm{M}$. After incubation, cells were washed with PBS 3 times. Coculture experiments were performed at a monocyte-to-fibroblast ratio of 4:1. Monocytes (500 $\times 10^{3}$ ) were added to a culture of $125 \times 10^{3}$ adventitial fibroblasts in $1.5 \mathrm{ml}$ of RPMI medium containing $5 \%$ FBS for 5 days. Cells in coculture were detached with $10 \mathrm{mM}$ EDTA/Accumax, centrifuged, and resuspended in PBS containing 0.5\% BSA, and they were kept on ice until they were analyzed by flow cytometry to detect the PE signal from PKH26 using a FACSCanto cytometer (BD Biosciences, San Jose, Calif., USA). For overnight storage, cells were resuspended in $0.5 \%$ paraformaldehyde-PBS solution, vortexed immediately, and then kept in the dark on a rotating holder at $4^{\circ} \mathrm{C}$.

\section{Animal Manipulations}

All mice were housed in the UTMB Animal Resource Center in accordance with its Institutional Animal Care and Use Committee guidelines, and the use of animals conformed to the Guide for the Care and Use of Laboratory Animals published by the US National Institutes of Health. Male C57BL/6J wild-type (WT) and $\mathrm{CCR}^{-/-}$mice (obtained as breeding pairs and bred in-house; C57BL/6J background) were purchased from Jackson Laboratory (Bar Harbor, Me., USA); they were maintained on a 12-hour light/ dark cycle and were allowed food and water ad libitum. CCR2null mice can produce MCP-1 but have cells lacking the ability to respond to this chemokine; therefore, they lack the CCR2+ monocyte-AoAF interaction. For Ang II infusion, anesthetized mice received subcutaneous osmotic minipumps (Alzet, Cupertino, Calif., USA) delivering either saline (sham) or Ang II (synthesized at $\mathrm{UTMB}$ ) at $2,500 \mathrm{ng} / \mathrm{kg} / \mathrm{min}$ for 10 days.

\section{Aortic Digestion and Flow Cytometric Quantitation of \\ Fibroblasts}

Aortas were removed from anesthetized mice, minced into 3to $4-\mathrm{mm}$ pieces, and placed in 1-ml digestion solution containing 0.6 units/ml Liberase Blendzyme 3 (Roche) and $50 \mu \mathrm{g} / \mathrm{ml}$ porcine pancreatic elastase (Sigma-Aldrich) in a base solution of DMEM. Aortic tissue was digested at $37^{\circ} \mathrm{C}$ with agitation for $1 \mathrm{~h}$. After digestion, cells were washed in FACS buffer ( $0.5 \%$ BSA and $0.02 \%$ $\mathrm{NaN}_{3}$ in DMEM) at $300 \mathrm{~g}$ for $5 \mathrm{~min}$ and then fixed and permeabilized in fixation/permeabilization solution (eBiosciences) for $30 \mathrm{~min}$ at $4^{\circ} \mathrm{C}$. Murine $\mathrm{Fc}$ receptors were blocked using antibodies against mouse CD16/32 antigens (eBioscience) for $10 \mathrm{~min}$ on ice. Fluorochrome-conjugated anti-ER-TR7 antibodies (eBioscience) were incubated for $30 \mathrm{~min}$ at $4^{\circ} \mathrm{C}$. Corresponding isotype control antibodies were added to 'isotype samples' at the same concentrations as the antibodies of interest. After incubation, samples were washed 3 times in FACS buffer, centrifuged at $300 \mathrm{~g}$ for $5 \mathrm{~min}$, and then fixed in $0.5 \%$ PFA and analyzed by FACSCanto. Debris and dead cells, defined by forward scatter, were excluded from analysis. Data were analyzed with FlowJo software.

\section{Monocyte Isolation}

Blood was obtained from WT C57BL/6J mice by cardiac puncture and erythrocytes lysed with RBC lysis solution (Invitrogen) added to the blood in a 3:1 ratio for $5 \mathrm{~min}$ at room temperature. Cells were centrifuged at $1,000 \mathrm{~g}$ for $3 \mathrm{~min}$ to remove the RBC lysis solution, and the leukocyte pellet was resuspended and washed in cell isolation buffer (2\% heat-inactivated FBS and 2 mM EDTA in PBS) followed by centrifugation at $400 \mathrm{~g}$ for $10 \mathrm{~min}$. Leukocyte counting was done using the Trypan blue exclusion method with a hemocytometer. Negative isolation of monocytes was performed according to the SpinSep Monocyte Isolation Custom Kit's instructions (Stem Cell Technologies, Vancouver, B.C., Canada). Briefly, an antibody cocktail was added to the leukocytes for $15 \mathrm{~min}$ at $4^{\circ} \mathrm{C}$ and then washed, and $250 \mu \mathrm{l}$ of SpinSep dense particles were added per $5 \times 10^{7}$ cells. This cell/particle suspension was incubated on ice for $20 \mathrm{~min}$ with occasional mixing. The mixture was gently layered over SpinSep density medium and then centrifuged. The enriched cells from the interface were carefully removed and washed, and PBS was added to resuspend the monocytes before injection. Eight mice allowed the isolation of $1.5 \times$ $10^{6}$ monocytes, representing $7 \%$ of all leukocytes. The purity was $>95 \%$ as assessed by staining for CD14. Monocytes from $\mathrm{CCR}^{-/-}$mice were obtained similarly.

\section{Adoptive Transfer of Mouse Monocytes}

Monocytes $\left(1.5 \times 10^{6}\right)$ in $150 \mu$ l of PBS were injected via the mouse tail vein using a 26-gauge needle; 4 days later, mice were sacrificed and the ascending aorta was embedded and quickly frozen in OCT on dry ice. Six-micrometer cross sections were cut and analyzed using Movat's stain. Micrographs were taken at a magnification of $20 \times$ with a Nikon Eclipse fluorescence microscope, and the images were collected using ACT-1 software. 
Table 1. Adventitial fibroblast and THP-1 monocytic cell cytokine secretion

\begin{tabular}{lcc}
\hline Cytokines/chemokines & AoAF & THP-1 \\
& pg/ml $\pm \mathrm{SD}$ & pg/ml $\pm \mathrm{SD}$ \\
\hline IL-1 $\beta$ & OOR & $71.8 \pm 12.4$ \\
IL-1Ra & $89.3 \pm 24.3$ & $33.3 \pm 17.4$ \\
IL-2 & OOR< & $10.8 \pm 4.0$ \\
IL-4 & $6.3 \pm 0.2$ & $5.9 \pm 0.3$ \\
IL-5 & $2.0 \pm 0.2$ & $1.4 \pm 0.1$ \\
IL-6 & $967.8 \pm 149.4$ & $3.4 \pm 0.9$ \\
IL-8 & $8.4 \pm 1.5$ & $21.2 \pm 5.2$ \\
IL-10 & $27.5 \pm 4.3$ & $12.7 \pm 0.7$ \\
IL-12p40/p70 & OOR< & $1.4 \pm 1.0$ \\
IL-15 & $15.3 \pm 2.5$ & $10.8 \pm 3.4$ \\
TNF- $\alpha$ & $3.2 \pm 0.7$ & $33.3 \pm 17.4$ \\
IFN- $\gamma$ & $2.4 \pm 0.3$ & $1.9 \pm 0.7$ \\
GM-CSF & $18.3 \pm 3.8$ & OOR< \\
MIP-1 $\alpha$ & $17.7 \pm 3.9$ & $20.7 \pm 5.0$ \\
MIP-1 $\beta$ & $18.1 \pm 4.9$ & $276.4 \pm 60.9$ \\
Eotaxin & $1.2 \pm 0.4$ & OOR< \\
RANTES & OOR< & $163.9 \pm 14.3$ \\
MCP-1 & $583.7 \pm 78.9$ & $19.7 \pm 3.1$ \\
IP-10 & $1.9 \pm 0.1$ & $8.2 \pm 1.2$ \\
\hline
\end{tabular}

Cytokines were secreted from AoAF and monocytes. AoAF $\left(50 \times 10^{3}\right)$ and THP-1 monocytes $\left(200 \times 10^{3}\right)$ were grown for 36 $h$, and cytokines in media were analyzed by multiplex ELISA. Data are means \pm SD for 6 samples of each cell type. OOR $<=$ Out of range low.

Quantitative Real-Time PCR

Total cellular RNA was extracted from AoAF using TRI Reagent (Sigma). Validated real-time PCR primers for human IL-6 (forward 5'-GGCACTGGCA GAAAACAACC-3' and reverse 5'-GCAAGTCTCCTCATTGAATCC-3') and MCP-1 (forward 5'CATTGTG GCCAAGGAGATCTG-3' and reverse 5'-CTTCGGAGTTTGGGTTTGCTT-3') were obtained from the RT Primer Database (http://medgen.ugent.be/rtprimerdb). Thermal cycling was performed with a BioRad MyIQ5 real-time PCR system (BioRad, Hercules, Calif., USA) under factory defaults. GAPDH mRNA levels were measured for each sample and utilized as the internal control. Fold change values were calculated using the $2^{-\Delta \Delta \mathrm{Ct}}$ method with the control sample as the calibrator.

\section{Immunohistochemistry}

Slides containing frozen cross sections $(6 \mu \mathrm{m})$ from Ang II- or sham-treated mouse aortas were fixed with $4 \%$ PFA for $30 \mathrm{~min}$, blocked with $0.3 \% \mathrm{H}_{2} \mathrm{O}_{2}$ for $10 \mathrm{~min}$ and $0.3 \%$ Triton-X and $5 \%$ goat serum for $1 \mathrm{~h}$ at room temperature, and then incubated with primary anti-fibroblast antibody (1:200, ER-TR7; Abcam) or antiPCNA $(1: 500 ; \mathrm{Abcam})$ at $4^{\circ} \mathrm{C}$ overnight. After washing, secondary antibodies were added at a dilution of 1:200 for $1 \mathrm{~h}$ at room temperature, followed by incubation with avidin-biotin complex for $1 \mathrm{~h}$ (Vector ABC kit). 3,3'-Diaminobenzidine (Vector) was ap- plied for $5 \mathrm{~min}$ to identify positive immunoreaction. Slides were counterstained with hematoxylin (for fibroblast imaging only), dehydrated in ethanol and xylene, and mounted in Cytoseal (Richard-Allan Scientific).

Data Analysis

Data are reported as the means \pm SD of 3 independent experiments unless otherwise noted. Differences were analyzed by Student's t test (2-tailed, assuming unequal variances), or by a 1-way analysis of variance (ANOVA) followed by a post hoc Tukey pairwise comparison. $\mathrm{p}<0.05$ was considered statistically significant.

\section{Results}

\section{Cytokine Production by Human AoAF}

While there has been growing interest in the role of adventitial fibroblasts in vascular pathology [21], the spectrum of cytokines produced by these cells has not been well described. Using a multiplex cytokine ELISA assay and SCGM bathing of AoAF for $36 \mathrm{~h}$, we tested the ability of AoAF to secrete constitutively numerous inflammatory cytokines. IL-6 and MCP-1 were detected at the highest concentration relative to all other cytokines which were detected at substantially lower concentrations (table 1). IL-6 and MCP-1 are important chemoattractants as well as cellular proliferation signals for monocytes and macrophages, and they are characteristic of Ang II-induced vascular inflammation. Of the cytokines detected, IL-1Ra, IL-6, IL-10, IL-15, and MCP-1 were measured at higher concentrations in AoAF than in THP1 monocytic cells grown at 4 times the cellular density in an equivalent tissue culture volume. GM-CSF and eotaxin were only detectable in AoAF cultures. In contrast, THP-1 cells produced higher concentrations of IL-8, MIP-1 $\beta$, TNF- $\alpha$, and IP-10. Furthermore, IL-1 $\beta$, IL-2, IL12 p40/p70, and RANTES were detectable only in THP-1 cultures.

\section{Ang II Increased Expression of IL-6 and MCP-1 in AoAF}

Since AoAF constitutively produced an abundant amount of IL- 6 and MCP-1 protein, we tested the ability of Ang II to induce their expression using quantitative real-time PCR. Ang II induced a rapid increase in IL-6 and MCP-1; IL-6 mRNA was induced 10-fold at $3 \mathrm{~h}$ and remained elevated at 4-6-fold up to $48 \mathrm{~h}$, while MCP-1 mRNA was induced 2 -fold at $3 \mathrm{~h}$ but had returned to baseline by $6 \mathrm{~h}$ (fig. 1). Together, these data indicate an Ang II-induced expression of monokines by AoAF. 


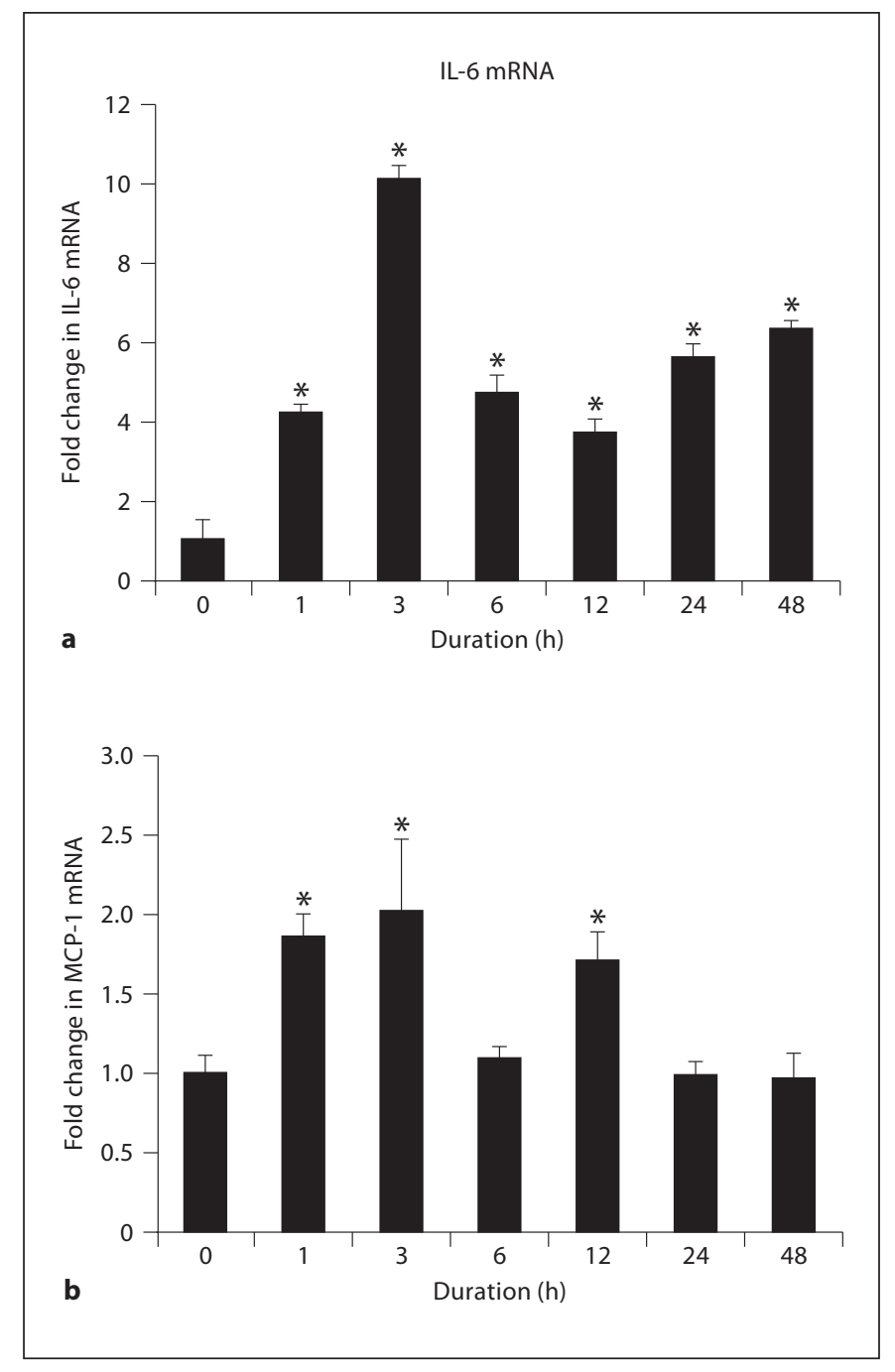

Fig. 1. Ang II induces increases in IL- 6 and MCP-1 mRNA in primary AoAF. The induction of IL-6 (a) and MCP-1 (b) mRNA levels after stimulation with $1 \mu \mathrm{M}$ Ang II from 0 to $48 \mathrm{~h}$ in tissue culture was measured by real-time PCR. Data shown are an average of 3 independent experiments. One-way ANOVA was significant at $\mathrm{p}<0.001$ for both IL- 6 and MCP- $1 .{ }^{*} \mathrm{p}<0.001$ for Tukey's post hoc pairwise comparison to $0 \mathrm{~h}$.

\section{MCP-1 Produced from AoAF-Recruited Monocytes}

To ensure that the MCP-1 produced by AoAF was functionally active, we performed cell migration assays using Boyden chambers with AoAF-conditioned medium as the attractant. There was a 3 -fold increase in the number of THP-1 cells that migrated toward the conditioned medium compared to the control media (fig. 2a). This effect was blocked dose dependently with an antiMCP-1 neutralizing antibody (58\% at the highest dose

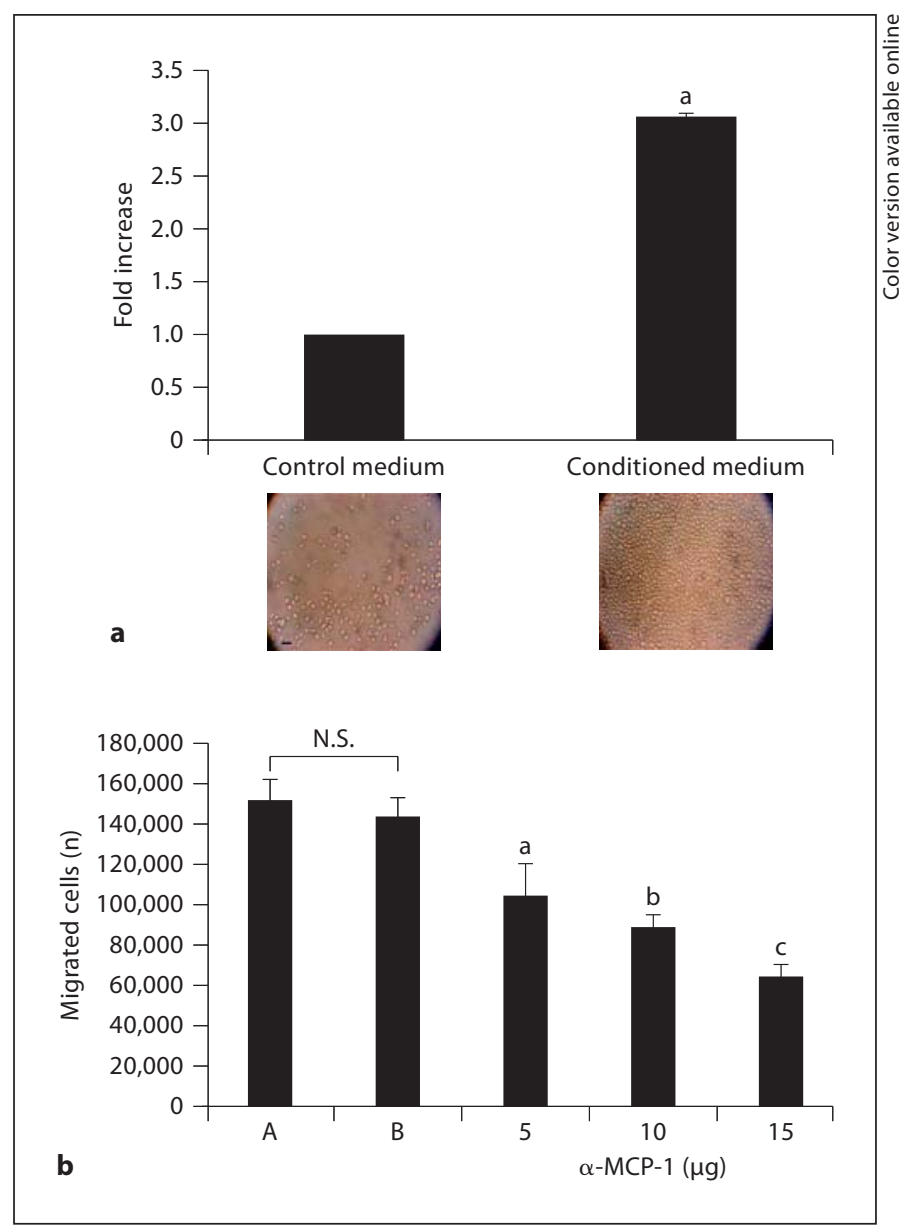

Fig. 2. MCP-1 produced by AoAF strongly recruits monocytes. The migration of THP-1 monocytes to AoAF-conditioned medium was measured by Boyden chamber migration assay. a Monocytes which had migrated to the bottom chamber (photo in a) after $2 \mathrm{~h}$ were quantitated using a Z2 Coulter Counter. b MCP-1mediated migration was assessed by the addition of neutralizing MCP-1 antibody. Anti-MCP-1 neutralizing antibody $(5,10$, and $15 \mu \mathrm{g}$ ) was used. $\mathrm{A}=$ Conditioned media; $\mathrm{B}=$ isotype antibody; N.S. = not significant $(\mathrm{p}=0.18) .{ }^{\mathrm{a}} \mathrm{p}<0.009 ;{ }^{\mathrm{b}} \mathrm{p}<0.001 ;{ }^{\mathrm{c}} \mathrm{p}<0.0003$ compared to the control. Data are means \pm SD. Microphotographs were taken at a magnification of $10 \times$. Scale bar $=40 \mu \mathrm{m}$.

used) (fig. 2b). These data indicate that AoAF secrete biologically active monocyte chemoattractant MCP-1.

\section{Ang II Stimulation as well as Coculturing AoAF with}

Monocytes Enhanced AoAF Proliferation

Ang II had a weak mitogenic effect on human AoAF measured as a slight but statistically significant increase in radiolabeled thymidine incorporation into AoAF (fig. 3a). Ang II exposure for a longer duration (4 days) 


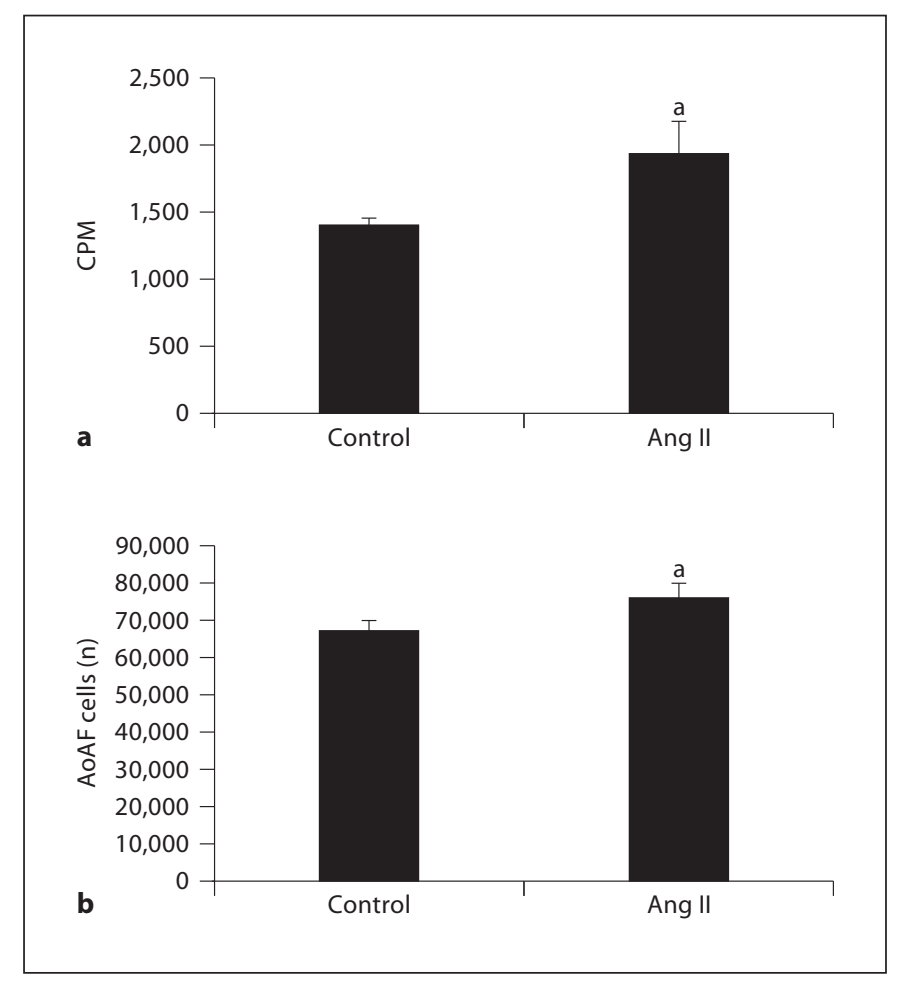

Fig. 3. AoAF respond to Ang II in tissue culture with proliferation. a AoAF were plated at $50 \times 10^{3}$ cells per well in 12 -well plates and grown in SCGM for $24 \mathrm{~h}$ prior to stimulation with $1 \mu \mathrm{M}$ Ang II for $16 \mathrm{~h} .{ }^{3} \mathrm{H}$-thymidine $(2 \mu \mathrm{Ci} / \mathrm{ml})$ was added during the final $4 \mathrm{~h}$. Cells were dissolved with $500 \mu \mathrm{l}$ of $0.2 \mathrm{~N} \mathrm{NaOH}$ and processed as detailed in Methods before counting in a Beckman Coulter scintillation counter. $\mathbf{b}$ The same experiment was performed, except the Ang II treatment lasted 4 days and cells were harvested and counted in a Z2 Coulter Counter. Data are the means \pm SD of 3 separate experiments. $\mathrm{CPM}={ }^{3} \mathrm{H}$ counts per minute. ${ }^{\mathrm{a}} \mathrm{p}<0.05$.

also produced a slight but significant increase in AoAF numbers (fig. 3b). Since monocytes/macrophages have been shown to be present in the adventitia of diseased vessels, we tested whether monocytes recruited by AoAF could stimulate the proliferation of AoAF. Coculturing AoAF and THP-1 cells led to a small but statistically significant increase in fibroblast cell number as compared to AoAF cultured alone over the same duration (fig. 4). To exclude potential monocyte contamination of the AoAF counting, we labeled the AoAF with PKH26, a PE dye, prior to flow cytometry (fig. 4a). The dye was not transferred to THP-1 cells (fig. $4 \mathrm{~b}$ ) and has been shown to pass only to daughter cells [22]. After coculture with unlabeled monocytes, labeled AoAF were detected as events in M1 (fig. 4c), and a small but significant increase was measured (fig. 4d).
Ang II Stimulated Adventitial Fibrosis in WT Mice

Staining for monocytes and macrophages previously demonstrated that the adventitia was the major site of macrophage recruitment after Ang II infusion in mice [7, $23,24]$. Coincidentally, the adventitia thickened mainly due to an increase in fibroblasts $[7,20]$. Immunohistochemical staining with the fibroblast-specific anti-ERTR7 antibody demonstrated aortic fibroblasts predominantly in the adventitia of control mice but also interspersed throughout the medial vessel wall (fig. 5a). A similar pattern was evident in aortas from mice infused with Ang II for 6 days, with strong staining of the adventitia which also demonstrated enhance cellularity (fig. 5b). PCNA staining was stronger in the adventitia of 6-day Ang II-infused mice versus sham mice, indicating the presence of increased numbers of proliferating cells in Ang II-treated mice (fig. 5c, d).

To quantitate the Ang II-induced stimulation of fibroblast proliferation in vivo, aortas from sham- and Ang II-stimulated mice were digested to obtain single cell preparations, and the fibroblast population was determined with flow cytometry using primary antifibroblast antibody ER-TR7 (Abcam). Figure 6a shows 2 representative flow cytometry experiments, and the quantitation from 3 experiments in figure $6 \mathrm{~b}$ demonstrates that Ang II induced a 3-fold increase in AoAF.

\section{Adoptive Transfer of CCR $2^{+/+}$Monocytes into}

CCR2-Deficient Mice Restored Adventitial Thickening

Increased fibrosis and expansion of adventitia has been reported to be blunted along with reduced macrophage recruitment to the aorta of CCR2-deficient mice infused with Ang II [25]. Since CCR2 is the receptor for MCP-1, we hypothesized that the recruitment of CCR $2^{+/+}$ monocytes and macrophages was a result of increased MCP-1 production by AoAF in response to Ang II, and that the increased monocyte recruitment was required for adventitial fibroblast proliferation leading to adventitial thickening. We performed an adoptive transfer of CCR $2^{+/+}$monocytes at a physiologically relevant number into Ang II-infused CCR $2^{--}$mice and found that the cellular content (red stain indicating cytoplasm) and thickness of the adventitia dramatically increased in the presence of CCR $2^{+/+}$monocytes (fig. 7). The increased adventitial thickening was similar to that observed in WT mice after 10 days of infusion. 


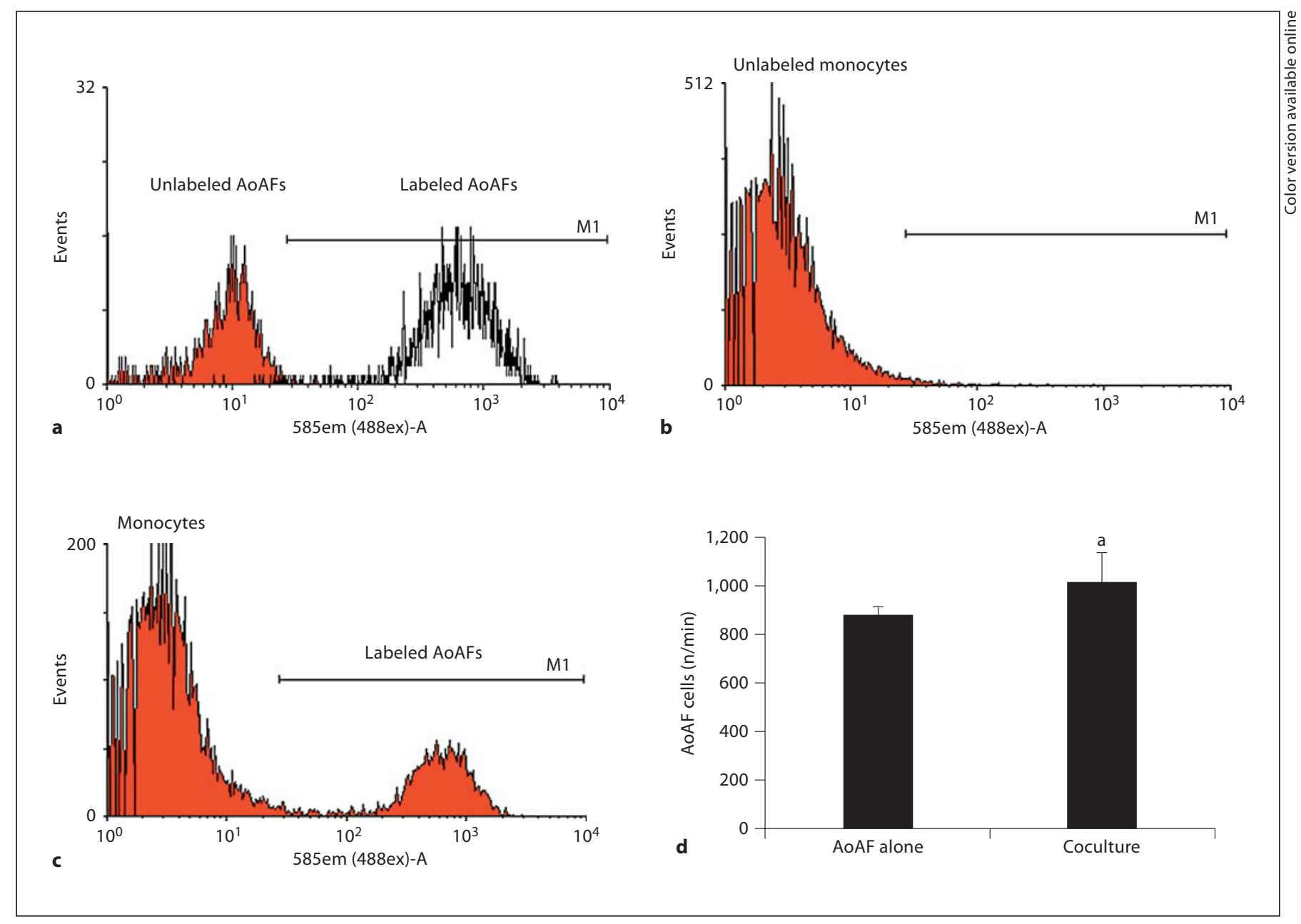

Fig. 4. Monocytes induce AoAF proliferation in coculture. a AoAF were labeled with PKH26 and detected by flow cytometry as events falling within region $\mathrm{Ml}$, based on isotype control staining (red curve; colors refer to the online version only). b THP-1 monocytes obtained after coculture for 4 days were checked for PKH26. c Labeled AoAF after coculture were detected as events in M1 and quantitated (d). ${ }^{a} \mathrm{p}<0.025$ compared to the number of AoAF in single culture. Data are mean $\pm \mathrm{SD}$.

\section{Discussion}

In this study, we used a combination of tissue culture and multiplex cytokine ELISA assays to demonstrate that AoAF produce and secrete numerous inflammatory cytokines and chemokines of relevance to vascular pathobiology, particularly IL-6 and MCP-1. We also showed that Ang II further upregulates the transcription of IL- 6 and MCP-1 in AoAF in vitro, and that MCP1 is the primary monocyte chemoattractant secreted by AoAF. Our finding that blocking MCP-1 activity with a neutralizing antibody significantly decreased, but did not totally block, monocyte recruitment suggests that additional chemokines produced by AoAF may also influence monocyte recruitment. While we identified numerous cytokines released from AoAF at relatively low concentrations in the tissue culture media, such results do not address their potential potency in vivo. Identifying these additional chemokines should provide a more thorough understanding of how monocytes/macrophages and other leukocytes are recruited into the adventitia. We also reported that THP-1 cells, a human leukemic promonocytic cell line, produced numerous cytokines in response to Ang-II, although their production of IL- 6 and MCP-1 was significantly less than the AoAF production of these factors. These cells have been 
Sham
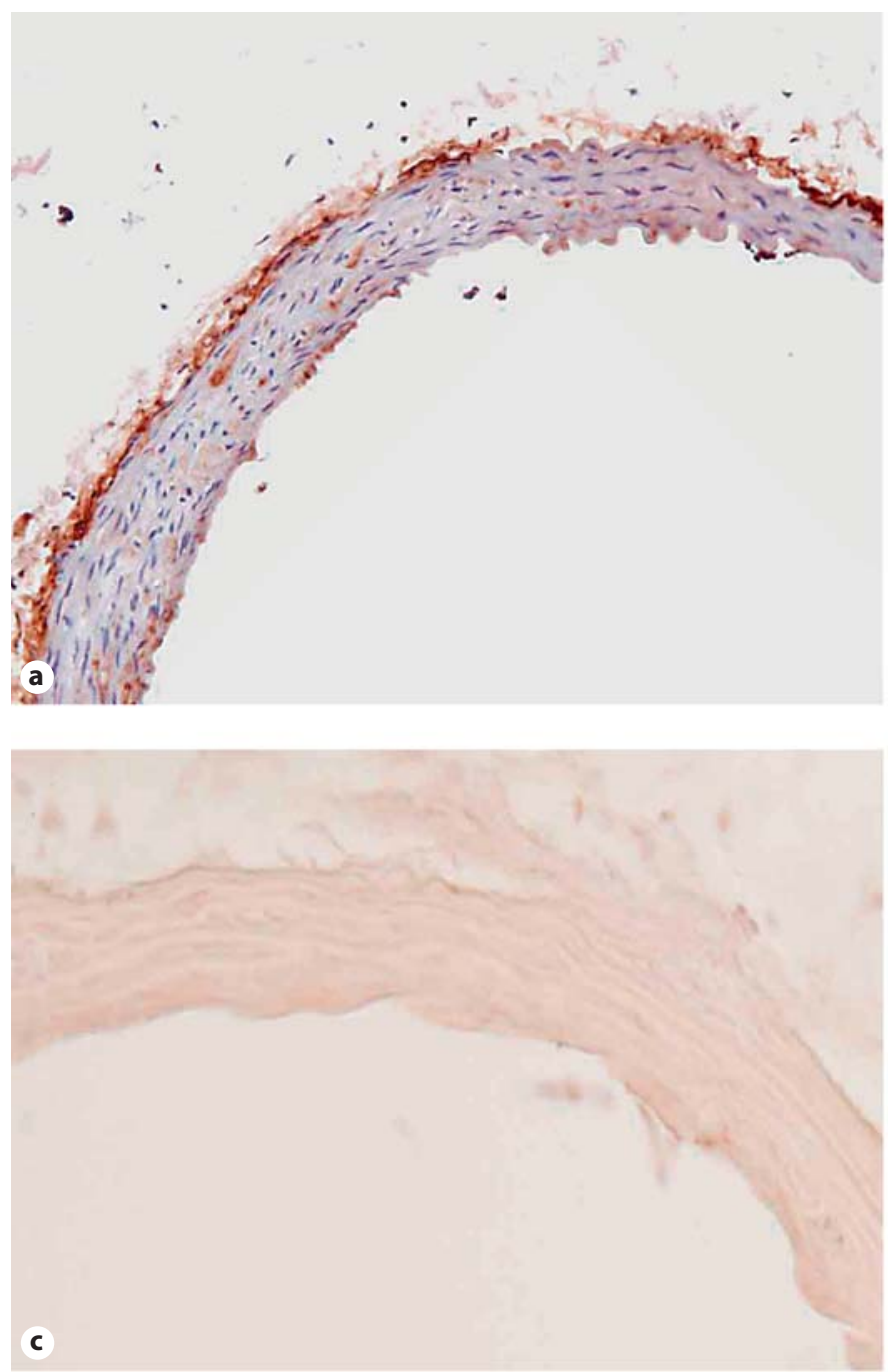

Fig. 5. Localization of $\operatorname{AoAF}(\mathbf{a}, \mathbf{b})$ and PCNA staining (c, d) in aorta from sham and Ang II-infused mice. Slides containing 6 - $\mu \mathrm{m}$ frozen cross sections of sham or Ang II-treated mouse aortas were fixed with $4 \%$ paraformaldehyde for $30 \mathrm{~min}$, exposed to primary and secondary antibodies as detailed in Methods, counterstained with hematoxylin (a and $\mathbf{b}$ only), and viewed with light microscopy at a magnification of $20 \times$. e Oil immersion magnification of the Ang II-stimulated aortic wall showing a higher magnification $(600 \times)$ of the expression of ER-TR7, presumably localized to fibroblasts.
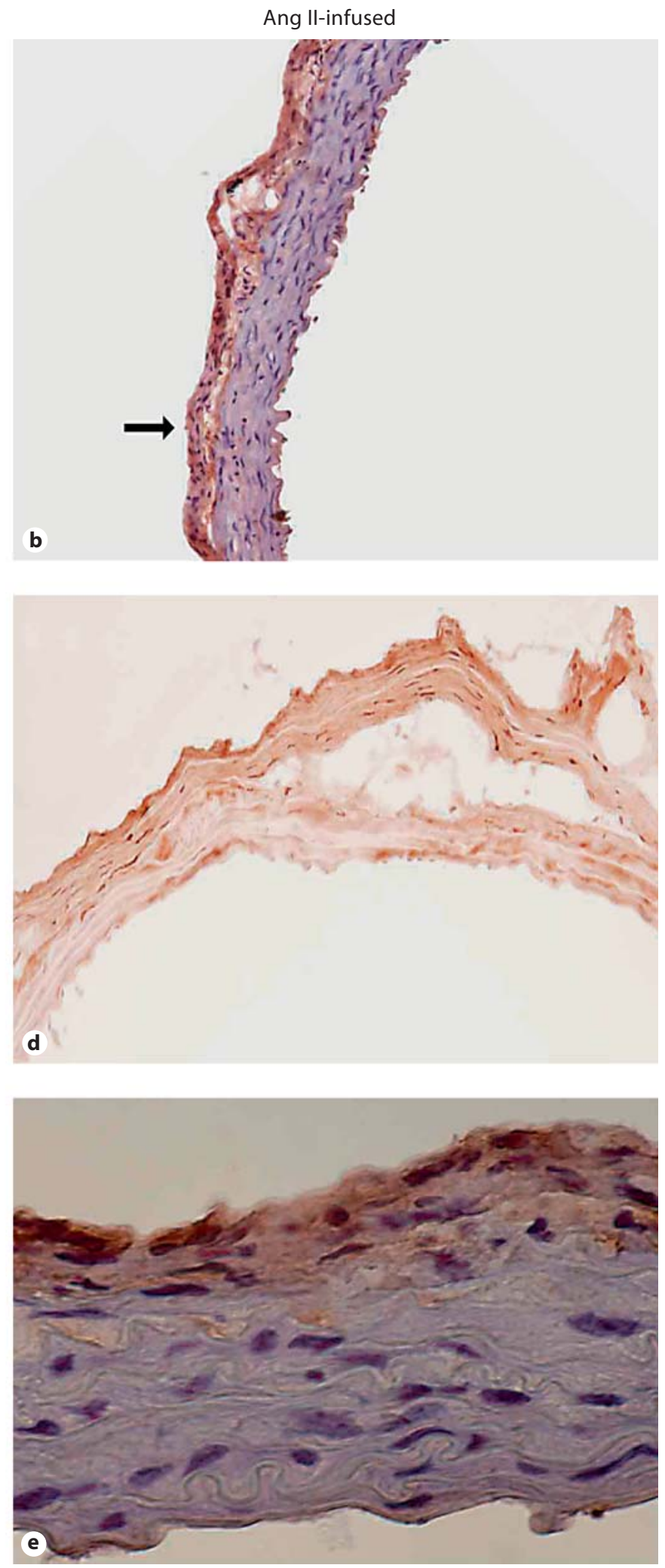

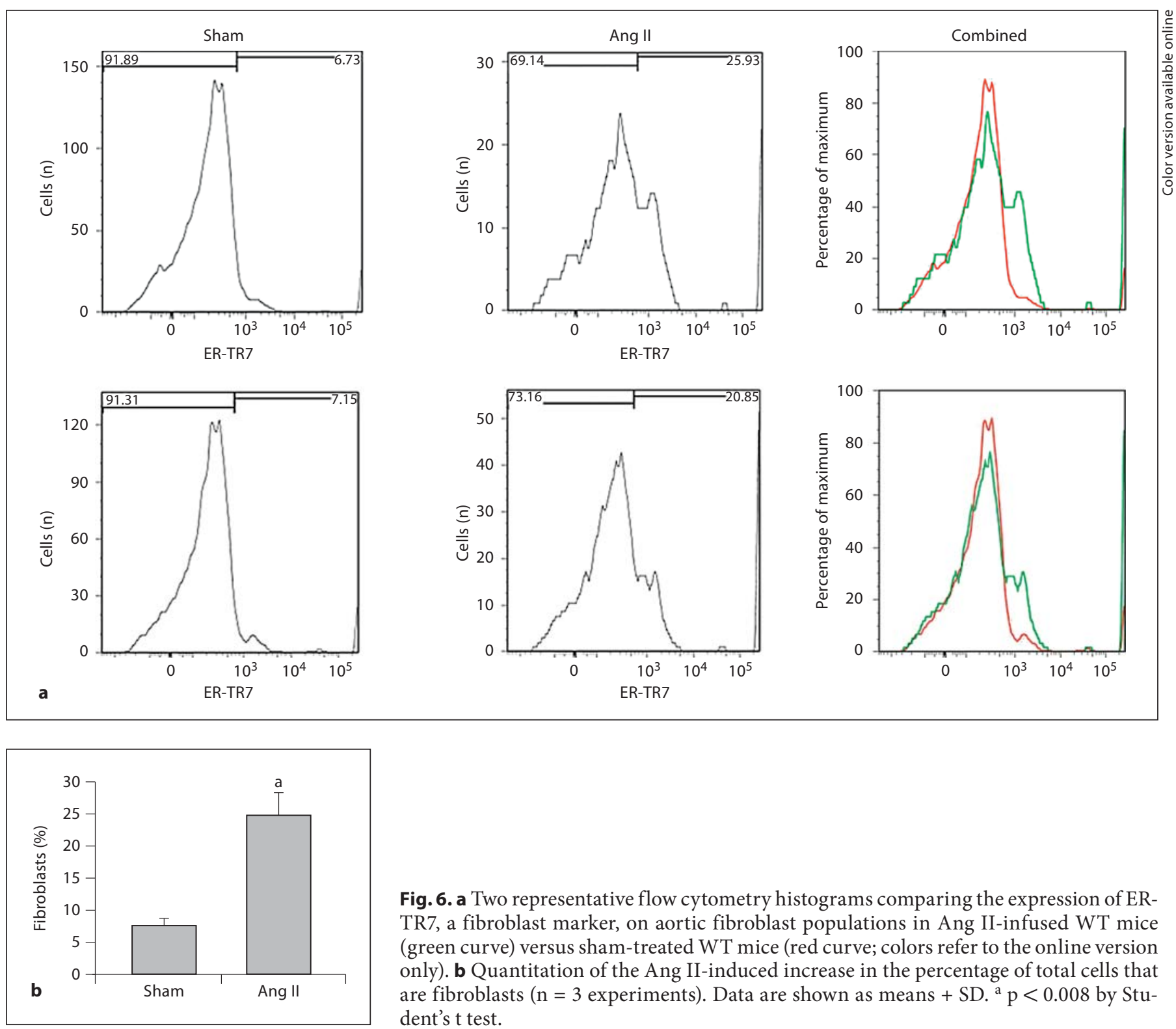

Fig. 6. a Two representative flow cytometry histograms comparing the expression of ERTR7, a fibroblast marker, on aortic fibroblast populations in Ang II-infused WT mice (green curve) versus sham-treated WT mice (red curve; colors refer to the online version only). b Quantitation of the Ang II-induced increase in the percentage of total cells that are fibroblasts ( $\mathrm{n}=3$ experiments). Data are shown as means + SD. ${ }^{\mathrm{a}} \mathrm{p}<0.008$ by Student's t test.

used extensively since they share many properties of primary monocytes, especially in terms of cytokine production and differentiation. Nevertheless, the fact that these cells are cancerous suggests that their production of factors responsible for cell growth and proliferation in vitro may differ significantly from primary monocytes.

Previous studies from our laboratory are consistent with the cytokine observations reported here. We have reported IL- 6 localization in the aortic wall of sham and Ang II-treated mouse aortas using in situ hybridization, and we have demonstrated a significant Ang II-induced increase in staining in the adventitia of these vessels [7]. In addition, we have measured cytokine production in time course experiments using adventitial explants stripped from the walls of sham- and Ang II-treated aortas, and we have reported significantly more IL- 6 cytokine production in the adventitial portion of the vessel wall than in the medial/endothelial layer [7].

Ang II infusion is a potent inducer of vascular inflammation, and its physiological effect has been extensively studied in mouse models. It is well established that Ang 


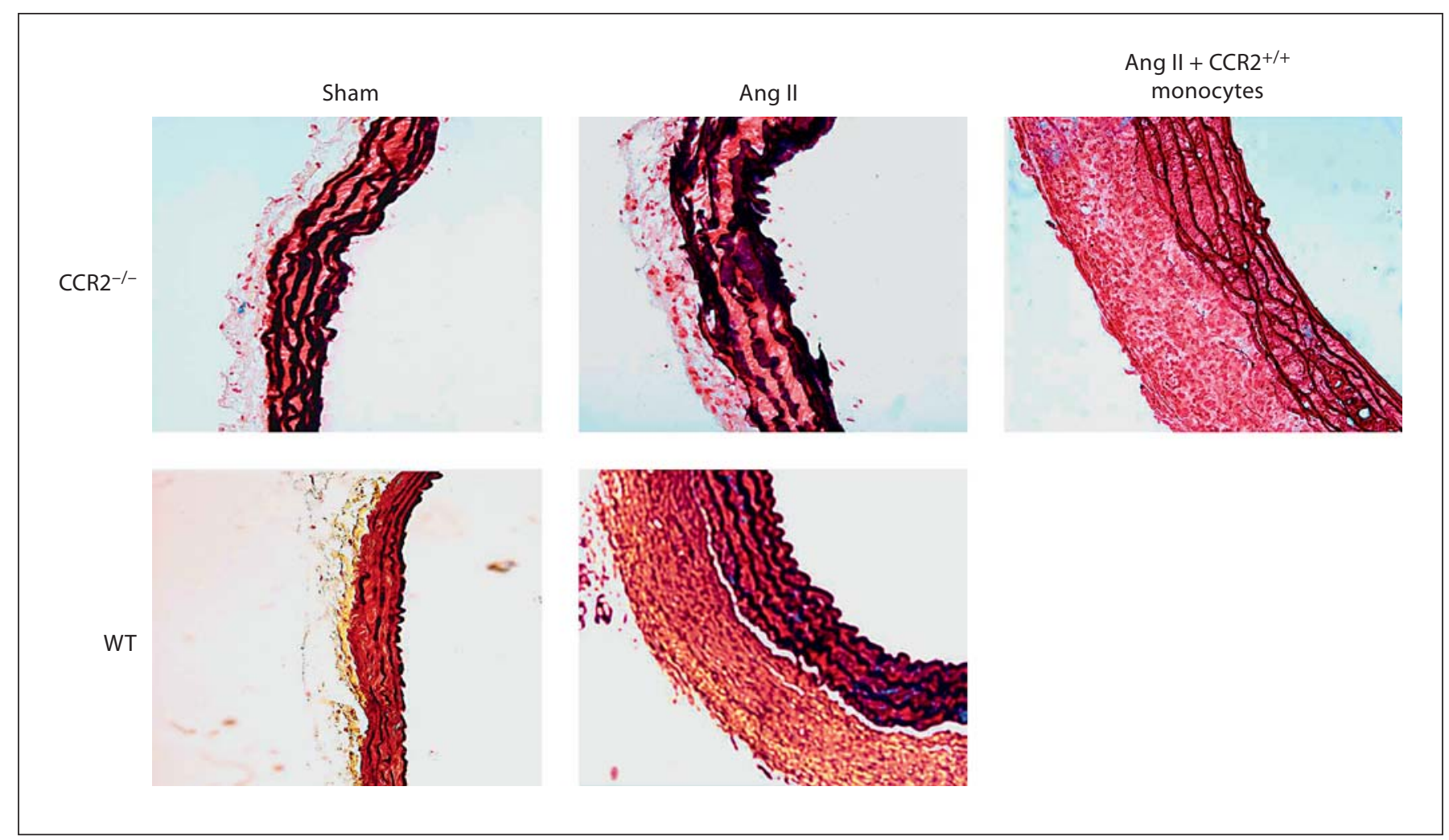

Fig. 7. CCR $2^{+/+}$monocytes increase adventitial fibroblast proliferation in vivo. CCR2 $2^{-/-}$and WT mice were infused with saline (sham) or Ang II for 10 days, and adventitial thickening in the ascending aorta was assessed by Movat's staining ( $\mathrm{n}=7$ in all 4 groups). Adoptive transfer of $1.5 \times 10^{6} \mathrm{CCR} 2^{+/+}$monocytes into sham-treated $(n=5)$ or Ang II-treated $(n=6) \mathrm{CCR}^{-/-}$mice via the tail vein was performed on day 6 of the Ang II infusion and the animals were sacrificed 4 days later. Cell/cytoplasm is red, collagen is yellow, elastin is black, and mucopolysaccharide is blue. Microphotographs of 6 - $\mu \mathrm{m}$-thick aortic cross sections were taken at a magnification of $20 \times$. Scale bar $=100 \mu \mathrm{m}$.
II infusion enhances macrophage recruitment and accelerates atherosclerosis and aneurysm formation [26-28], and monocyte chemokines including osteopontin and MCP-1 have been implicated. However, many of these studies have been limited by the inability of histochemistry methods to systematically quantitate regional differences in macrophage number as well as their activation state. Previously, we had adapted methods of aortic tissue dissociation and quantitative flow cytometry to demonstrate that Ang II potently induced monocyte-to-macrophage activation and dramatically increased macrophage numbers in animals with aortic dilatation and dissection [29].

Because AoAF can recruit monocytes, and since macrophages are found in the adventitia of human and rodent atherosclerotic vessels and aortic aneurysms [20, 30, 31], we had hypothesized that monocytes contribute to fibroblast proliferation. Using CCR $2^{-/-}$mice, we demonstrat- ed less adventitial thickening in response to Ang II than in WT mice; using adoptive transfer of CCR $2^{+/+}$monocytes into the CCR2-null mice, we showed that recruitment of CCR $2^{+/+}$monocytes restored the adventitial thickening in response to the mitogenic effect of Ang II. We have previously identified, using the same adoptive transfer of fluorescently labeled monocytes, 2 regional sites within the aorta for the uptake and adventitial accumulation of monocytes that were dependent on Ang II stimulation and CCR2 signaling [29]. In that study, we demonstrated that monocyte recruitment into the aorta potentiated IL- 6 production, that it was predominantly localized in the adventitia of our animal model, and that this process was governed by paracrine factors released during monocyte-aortic fibroblast interactions. Although the adventitial thickening in this study may be partly mediated by infiltrating leukocytes, we have previously shown that adventitial fibroblasts comprise most of 
the thickened adventitia in response to Ang II infusion in mice [7]. That observation, based on immunofluorescent staining of adventitial fibroblasts, was confirmed here as well. In this study, using quantitative flow cytometry on single cell preparations from enzymatically digested aortic tissue, we demonstrate that Ang II significantly increased the number of recoverable fibroblasts from the vessel wall. In addition, we showed increased PCNA staining of the adventitia from Ang II-treated versus control aortas. While the latter observation correlates with increased fibroblast staining, we have not definitively shown that the increased PCNA staining is within the proliferating fibroblasts. In addition, our studies do not preclude the possibility of fibroblast migration contributing to the increased numbers of adventitial fibroblasts. Nevertheless, taken together, we have provided strong documentation of Ang II-induced increases in adventitial fibroblast proliferation. While we have not yet identified how monocytes induce further AoAF proliferation, several secreted cytokines from monocytes are known to induce proliferation, including IL-1, TNF, p43, and substances similar to PDGF [32, 33]. Interestingly, we showed that IL- 1 and TNF are secreted by THP-1 monocytes in tissue culture at greater levels than adventitial fibroblasts (table 1).
In conclusion, our data strongly indicate that aortic adventitial fibroblasts are key mediators of vascular wall inflammation. These cells respond to Ang II stimulation by proliferating and producing cytokines and chemokines that can recruit monocytes and other leukocytes such as $\mathrm{T}$ and $\mathrm{B}$ lymphocytes. The monocyte-adventitial fibroblast interaction further increases AoAF cell proliferation, leading to further cytokine production, leukocyte recruitment, and expansion of the adventitia, 3 hallmarks of adventitial inflammation seen in cardiovascular diseases such as inflammatory aortic aneurysm and advanced atherosclerosis. This series of events may contribute significantly to the pathophysiology of the aortic wall which leads to an increased risk of plaque rupture in atherosclerotic vessels with extensive adventitial inflammation.

\section{Acknowledgements}

We would like to acknowledge the Histopathology and Flow Cytometry Core Facilities at UTMB, as well as Dr. Stefan Serabyn for synthesizing angiotensin II. This work was supported by the National Institutes of Health (DK079053 to R.G.T., P50 HL083794 and HL70925 to A.R.B., and T32ES007254 to B.C.T.) and by the James W. McLaughlin Fellowship Fund (B.C.T.). Core laboratory support was provided by P30 ES06676 to J.H., UTMB, and BAAHL-02-04 to A.K., UTMB.

\section{References}

1 Haurani MJ, Pagano PJ: Adventitial fibroblast reactive oxygen species as autacrine and paracrine mediators of remodeling: bellwether for vascular disease? Cardiovasc Res 2007;75:679-689.

-2 Pagano PJ, Clark JK, Cifuentes-Pagano ME, Clark SM, Callis GM, Quinn MT: Localization of a constitutively active, phagocyte-like NADPH oxidase in rabbit aortic adventitia: enhancement by angiotensin II. Proc Natl Acad Sci USA 1997;94:14483-14488.

$\checkmark 3$ Shen WL, Gao PJ, Che ZQ, Ji KD, Yin M, Yan C, Berk BC, Zhu DL: NAD(P)H oxidase-derived reactive oxygen species regulate angiotensin-II induced adventitial fibroblast phenotypic differentiation. Biochem Biophys Res Commun 2006;339:337-343.

4 Li G, Chen SJ, Oparil S, Chen YF, Thompson JA: Direct in vivo evidence demonstrating neointimal migration of adventitial fibroblasts after balloon injury of rat carotid arteries. Circulation 2000;101:1362-1365.

5 Sartore S, Chiavegato A, Faggin E, Franch R, Puato M, Ausoni S, Pauletto P: Contribution of adventitial fibroblasts to neointima formation and vascular remodeling: from innocent bystander to active participant. Circ Res 2001;89:1111-1121.
-6 Siow RC, Mallawaarachchi CM, Weissberg PL: Migration of adventitial myofibroblasts following vascular balloon injury: insights from in vivo gene transfer to rat carotid arteries. Cardiovasc Res 2003;59:212-221.

$\checkmark 7$ Recinos A 3rd, LeJeune WS, Sun H, Lee CY, Tieu BC, Lu M, Hou T, Boldogh I, Tilton RG, Brasier AR: Angiotensin II induces IL-6 expression and the Jak-STAT3 pathway in aortic adventitia of LDL receptor-deficient mice. Atherosclerosis 2007;194:125-133.

$\checkmark 8$ Xu F, Ji J, Li L, Chen R, Hu W-C: Adventitial fibroblasts are activated in the early stages of atherosclerosis in the apolipoprotein E knockout mouse. Biochem Biophys Res Commun 2007;352:681-688.

$\checkmark 9$ Rayner K, Van ES, Groot PH, Reape TJ: Localisation of mRNA for JE/MCP-1 and its receptor CCR2 in atherosclerotic lesions of the ApoE knockout mouse. J Vasc Res 2000;37: 93-102.

$>10$ Maiellaro K, Taylor WR: The role of the adventitia in vascular inflammation. Cardiovasc Res 2007;75:640-648.

11 Albutt C: Diseases of the Arteries Including Angina Pectoris. London, Macmillan, 1915.
12 Kohchi K, Takebayashi S, Hiroki T, Nobuyoshi M: Significance of adventitial inflammation of the coronary artery in patients with unstable angina: results at autopsy. Circulation 1985;71:709-716.

13 Laine P, Kaartinen M, Penttila A, Panula P, Paavonen T, Kovanen PT: Association between myocardial infarction and the mast cells in the adventitia of the infarct-related coronary artery. Circulation 1999;99:361369.

14 Wallsh E, Weinstein G, Franzone A, Clavel A, Rossi P, Kreps E: Inflammation of the coronary arteries in patients with unstable angina. Tex Heart Inst J 1986;13:105-108.

$\checkmark 15$ Higuchi ML, Gutierrez PS, Bezerra HG, Palomino SA, Aiello VD, Silvestre JM, Libby P, Ramires JA: Comparison between adventitial and intimal inflammation of ruptured and nonruptured atherosclerotic plaques in human coronary arteries. Arq Bras Cardiol 2002;79:20-24

16 Moreno PR, Purushothaman KR, Fuster $\mathrm{V}$, O'Connor WN: Intimomedial interface damage and adventitial inflammation is increased beneath disrupted atherosclerosis in the aorta: implications for plaque vulnerability. Circulation 2002;105:2504-2511. 
-17 Schwartz CJ, Mitchell JR: Cellular infiltration of the human arterial adventitia associated with atheromatous plaques. Circulation 1962;26:73-78.

18 Ramshaw AL, Roskell DE, Parums DV: Cytokine gene expression in aortic adventitial inflammation associated with advanced atherosclerosis (chronic periaortitis). J Clin Pathol 1994;47:721-727.

19 Michel JB, Thaunat O, Houard X, Meilhac O, Caligiuri G, Nicoletti A: Topological determinants and consequences of adventitial responses to arterial wall injury. Arterioscler Thromb Vasc Biol 2007;27:1259-1268.

-20 Sakata N, Nabeshima K, Iwasaki H, Tashiro T, Uesugi N, Nakashima O, Ito H, Kawanami T, Furuya K, Kojima M: Possible involvement of myofibroblasts in the development of inflammatory aortic aneurysm. Pathol Res Pract 2007;203:21-29.

21 Maiellaro K, Taylor WR: The role of the adventitia in vascular inflammation. Cardiovasc Res 2007;75:640-648.

-22 Johnsson C, Festin R, Tufveson G, Totterman TH: Ex vivo PKH26-labelling of lymphocytes for studies of cell migration in vivo. Scand J Immunol 1997;45:511-514.
23 Daugherty A, Rateri DL, Cassis LA: Role of the renin-angiotensin system in the development of abdominal aortic aneurysms in animals and humans. Ann NY Acad Sci 2006; 1085:82-91.

24 Deng G, Martin-McNulty B, Sukovich DA, Freay A, Halks-Miller M, Thinnes T, Loskutoff DJ, Carmeliet P, Dole WP, Wang YX: Urokinase-type plasminogen activator plays a critical role in angiotensin II-induced abdominal aortic aneurysm. Circ Res 2003;92: 510-517.

25 Ishibashi M, Hiasa K, Zhao Q, Inoue S, Ohtani K, Kitamoto S, Tsuchihashi M, Sugaya T, Charo IF, Kura S, Tsuzuki T, Ishibashi T, Takeshita A, Egashira K: Critical role of monocyte chemoattractant protein-1 receptor CCR2 on monocytes in hypertensioninduced vascular inflammation and remodeling. Circ Res 2004;94:1203-1210.

26 Daugherty A, Cassis L: Angiotensin II and abdominal aortic aneurysms. Curr Hypertens Rep 2004;6:442-446.

27 Saraff K, Babamusta F, Cassis LA, Daugherty A: Aortic dissection precedes formation of aneurysms and atherosclerosis in angiotensin II-infused, apolipoprotein E-deficient mice. Arterioscler Thromb Vasc Biol 2003; 23:1621-1626.

-28 Lu H, Rateri DL, Cassis LA, Daugherty A The role of the renin-angiotensin system in aortic aneurysmal diseases. Curr Hypertens Rep 2008;10:99-106.
29 Tieu BC, Lee C, Sun H, LeJeune W, Recinos A 3rd, Ju X, Spratt H, Guo DC, Milewicz D, Tilton RG, Brasier AR: An adventitial IL-6/ MCP1 amplification loop accelerates macrophage-mediated vascular inflammation leading to aortic dissection in mice. J Clin Invest 2009;119:3637-3651.

30 Daugherty A, Manning MW, Cassis LA: Angiotensin II promotes atherosclerotic lesions and aneurysms in apolipoprotein E-deficient mice. J Clin Invest 2000;105:16051612.

31 Saraff K, Babamusta F, Cassis LA, Daugherty A: Aortic dissection precedes formation of aneurysms and atherosclerosis in angiotensin II-infused, apolipoprotein E-deficient mice. Arterioscler Thromb Vasc Biol 2003; 23:1621-1626.

$>32$ Austgulen R, Hammerstrom J, Nissen-Meyer J: In vitro cultured human monocytes release fibroblast proliferation factor(s) different from interleukin 1. J Leukoc Biol 1987;42: $1-8$.

33 Park SG, Shin H, Shin YK, Lee Y, Choi EC, Park BJ, Kim S: The novel cytokine p43 stimulates dermal fibroblast proliferation and wound repair. Am J Pathol 2005;166:387398. 